\title{
On biases in Precise Point Positioning with multi-constellation and multi-frequency GNSS data
}

\author{
A El-Mowafy $^{1 *}, \mathrm{M} \mathrm{Deo}^{2}$ and C Rizos ${ }^{3}$ \\ ${ }^{1}$ Department of Spatial Sciences, Curtin University, GPO Box U 1987, Perth WA 6845, Australia \\ ${ }^{2}$ Air Services Australia, Canberra, Australia. \\ ${ }^{3}$ School of Civil and Environmental Engineering, University of New South Wales, Kensington, NSW 2052, Australia. \\ *a.El-Mowafy@curtin.edu.au, Tel: +61892663403, Fax: +61892662703
}

\begin{abstract}
Various types of biases in Global Navigation Satellite Systems (GNSS) data preclude integer ambiguity fixing and degrade solution accuracy when not being corrected during precise point positioning (PPP). In this contribution, these biases are first reviewed, including satellite and receiver hardware biases, differential code biases, differential phase biases, initial fractional phase biases, inter-system receiver time biases and system time scale offset. PPP models that take account of these biases are presented for two cases using ionosphere-free observations. The first case is when using primary signals that are used to generate the precise orbits and clock corrections. The second case applies when using signals additional signals to the primary ones. In both cases, measurements from a single and multiple-constellations are addressed. It is suggested that the satellite-related code biases be handled as calibrated quantities that are obtained from Multi-GNSS Experiment (MGEX) products and the fractional phase cycle biases obtained from a network to allow for integer ambiguity fixing. Some receiver-related biases are removed using between-satellite single-differencing, whereas other receiver biases such as inter-system biases are lumped with differential code and phase biases and need to be estimated. Testing results show that treatment of biases significantly improves solution convergence in the float ambiguity PPP mode and leads to ambiguity-fixed PPP within a few minutes with a small improvement of solution precision.
\end{abstract}

Keywords: GNSS, precise point positioning, biases, GPS.

\section{Introduction}

In PPP, it is crucial to account for all biases during measurement processing to achieve high accuracy. For instance, the use of the International GNSS Service (IGS) clock corrections computed from ionosphere-free observations that has embedded differential code biases introduces these biases in the phase observations. Such biases, in addition to biases of phase observations, propagate with the phase ambiguities. Hence, proper treatment of these biases will allow integer ambiguity resolution in PPP, leading to an overall improvement in positioning performance. Such treatment has to consider that not all biases can be estimated as additional unknowns in the PPP model along with the position, phase ambiguities and atmospheric parameters. This is mainly due to that different biases, if considered individually, are identically modelled, resulting in a rank deficiency. Thus, one strategy to handle biases is to take advantage of the relatively stable nature of satellite related biases and estimates these biases externally using reference stations and provide them to users to calibrate them out. At the receiver end, the use of between satellites single-differences (BSSD) is an effective approach to cancel receiver-related biases from the same frequency band from satellites that belong to the same constellation.

Ge et al (2008) have discussed estimation of the satellite single-differenced phase biases using a ground tracking network. The estimation of phase biases was affected by code biases. An alternate wide-lane phase bias calibration method is proposed in Banville et al (2008). Collins et al (2010) merged code and phase biases separately with clock offsets in a decoupled clock model. A similar model is presented in Laurichesse et al $(2008,2009,2015)$ using 'phase clocks', assuming that code biases are known. Wen et al (2011) proposed estimation of geometry-free receiver and satellite phase biases as additional parameters in Kalman filtering and sequentially fixing undifferenced ambiguities. Khodabandeh and Teunissen (2014) discussed

Measurement Science and Technology, Vol. 27, No. 3, 035102. DOI: 10.1088/0957-0233/27/3/035102

Page 1 
computing differential phase biases (DPBs) combined with other biases using an array of antennas separated by very short distances to reduce the code-dominant noise and multipath errors. Wübbena et al (2014) discussed exchange of satellite phase bias data using the Radio Technical Commission for Maritime Services (RTCM)-State Space Representation (SSR) format, message types (1265-1270) for different constellations in uncombined form to allow for implementation within different methods of ambiguity resolution. Laurichesse (2015) showed successful implementation of the individual satellite phase biases using multi-frequency scenarios.

Compared with the traditional use of dual-frequency single constellation data in PPP, the use of multifrequency and multi-constellation data offers several advantages. These advantages include improved accuracy and precision, and reduction of convergence time. For instance, Cai (2009) integrated Global Positioning System (GPS) and Global Navigation Satellite System (GLONASS) measurements into a PPP algorithm and reported an improvement of about $24 \%$ in positional accuracy. Similar improvements were achieved by Li et al (2013) when combining GPS and BeiDou in PPP. However, when integrating measurements from multiple constellations additional biases such as inter-system receiver time biases (ISB) and time scale offsets (ISTB) are encountered.

In this contribution we revisit the topic of dealing with biases in PPP using multi-constellation GNSS data motivated by the following. Firstly, most literature introduces biases in a combined form with little detail and focus only on the use of dual-frequency observations. In this manuscript, we provide a more insight into source and presentation of biases that would be useful for other investigations. Secondly, when users implement biases that are externally provided from a service network, we highlight the interoperability issue where a user should utilize measurements that are compatible with the ones used at the service network to generate these biases. Therefore, this paper aims to clarify different types of biases that are involved when using different possible measurement combinations, particularly when integrating data from multiple constellations and frequencies. In addition, the paper discusses sources freely available to compensate for some of these biases.

The characteristics of our strategy in this study to deal with biases in PPP, targeting integer-ambiguity fixing, includes the following i) use of the precise orbits, clock corrections and differential code biases that are generated by the IGS Multi-GNSS Experiment (MGEX); ii) the bias terms are reparametrized; iii) fractional phase cycle biases are assumed computed from a reference network; iv) BSSD observations are used; and finally v) ionosphere-free observations from single or multiple GNSS constellations are considered.

The remainder of this paper is organized as follows. The next section reviews the different types of measurement biases that affect single and multi-constellation PPP. Several PPP models are discussed in the following section that provides a rigorous treatment of biases. Modelling options for these biases in the more challenging case of multi-frequency, multi-constellation GNSS data is presented. Results and conclusions are presented thereafter.

\section{Biases in Single GNSS constellation observation equations}

For the PPP model, where precise orbits and clock corrections are used, the observation equations for pseudorange code and phase measurements for satellite $k$ from a GNSS constellation, such as GPS (denoted here as $G$ ), to receiver $r$ for signal $c_{i}$ on frequency $i$ in length units can be formulated as:

$$
\begin{aligned}
P\left(c_{i}\right)_{r}^{k_{G}}= & \rho_{r}^{k_{G}}+c\left(d t_{r_{G}}-d t^{k_{G}}+d_{r_{G}}\left(c_{i}\right)-d^{k_{G}}\left(c_{i}\right)\right)+T^{k_{G}}+\mu_{i} I^{k_{G}}+\varepsilon_{P\left(c_{i}\right)_{r}^{k_{G}}} \\
\phi\left(c_{i}\right)_{r}^{k_{G}}= & \rho_{r}^{k_{G}}+c\left(d t_{r_{G}}-d t^{k_{G}}\right)+\delta_{r_{G}}\left(c_{i}\right)-\delta^{k_{G}}\left(c_{i}\right)+T^{k_{G}}+\lambda_{i}\left(N_{r}^{k_{G}}\left(c_{i}\right)+\operatorname{IFPB}\left(c_{i}\right)-\mu_{i} I^{k_{G}}+\right. \\
& \varepsilon_{\phi\left(c_{i}\right)_{r}^{k_{G}}}
\end{aligned}
$$

where $P\left(c_{i}\right)_{r}^{k_{G}}$ and $\phi\left(c_{i}\right)_{r}^{k_{G}}$ denote code and phase measurements, respectively; $\rho_{r}^{k_{G}}$ is the satellite-toreceiver geometric range; $c$ is the speed of light in vacuum; $d t_{r_{G}}$ and $d t^{k_{G}}$ are the receiver and satellite clock offsets, where the latter is eliminated in PPP by the use of clock corrections. $T^{k_{G}}$ is the troposphere 
delay modelled as one vertical component for all satellites projected along the receiver-to-satellite direction using a mapping function (Tuka and El-Mowafy 2013); $\lambda_{i}$ denotes the wavelength for frequency $i ; \mu_{i}=\frac{f_{1}^{2}}{f_{i}^{2}}$ is the dispersive coefficient of the ionosphere; $I$ is the ionosphere error for a reference frequency, e.g. L1 for GPS, and $N_{r}^{k_{G}}\left(c_{i}\right)$ is the integer ambiguity. $\varepsilon_{\phi\left(c_{i}\right)_{r}^{k_{G}}}$ and $\varepsilon_{P\left(c_{i}\right)_{r}^{k_{G}}}$ include measurement noise and multipath of phase and code measurements. $d_{r_{G}}\left(c_{i}\right)$ and $d^{k_{G}}\left(c_{i}\right)$ are the receiver and satellite hardware biases in time units for code measurement, respectively. $\delta_{r_{G}}\left(c_{i}\right)$ and $\delta^{k_{G}}\left(c_{i}\right)$ are the receiver and satellite hardware biases for phase observation in length units. It is assumed that the receiver hardware biases $\delta_{r_{G}}\left(c_{i}\right)$ and $d_{r_{G}}\left(c_{i}\right)$ are the same for measurements of the same frequency for all satellites from the same constellation.

$I F P B$ denotes the geometric initial fractional phase cycle bias. It may exist due to the receiver tracking method and represents the geometric difference between the measured phase (after compensation for ambiguities and excluding other biases) and the actual receiver-to-satellite range at the receiver initialization (switch-on). In equation (2), IFPB is not merged with the hardware delay as they differ in the sense that $I F P B$ is constant for each session as long as the receiver keeps tracking the satellite and it may change with a switch-on and off of the receiver, whereas the phase hardware delay is stable and very slowly changes with time. However, in practice $I F P B$ is inseparable from the hardware delay, and we do not attempt to do that here as will be clarified later. For a single receiver, $I F P B$ is frequency dependent, and it is assumed the same for measurements on the same frequency for all satellites from the same constellation.

In PPP, users mostly employ IGS corrections to clock offsets that are computed from an ionosphere-free combination of primary signals. They may additionally employ measurement combinations of other signals as well as measurements from multiple constellations. The next section discusses different types of biases that are present in these cases and the following section will discuss parametrization of these biases.

\section{Types of Biases}

This section introduces different types of biases that affect multi-frequency single and multi-constellation GNSS measurements. The inter-frequency channel biases of GLONASS measurements and the quarter-cycle phase shifts between the in-phase and quadrature signals will not be covered here as the former were discussed in Reussner and Wanninger (2011); Aggrey and Bisnath (2014), and the latter were discussed in Wübbena et al (2009).

\subsection{Inter-system time biases (ISTB)}

Measurements from each GNSS constellation have satellite clock offsets that are referenced to its time scale. To account for this inter-system time offsets, two methods can be used. The first is to estimate it as an independent parameter for each system; while the second is to estimate the clock offset for one system and then estimate the time differences for the other systems relative to the reference one. These are defined as inter-system time offset (biases) - (ISTBs), which need to be modelled as extra parameters in PPP. This parameter can be combined into one parameter with the receiver time offset (ISB) as will be discussed in section 3.5. In the future, precise clock corrections for the new constellations generated by MGEX will be referenced to GPS time scale (Steigenberger et al 2014). Hence, there will be no need to consider ISTBs when using MGEX products.

\subsection{Hardware biases}

The receiver and satellite hardware biases $\left(d_{r_{G}}, \delta_{r_{G}}\right)$ and $\left(d^{k_{G}}, \delta^{k_{G}}\right)$ are caused by several sources including digital delays in the signal generator, signal distortion, the processing filters, correlator differences handling signal modulation, firmware biases, bandwidth dissimilarities, in addition to signal path through the antenna, splitter, cabling and amplifier (Phelts 2007). Moreover, hardware biases may exist due to incoherence (misalignment) between the signal code and phase signals. Such an effect has been observed on the L5 signals of the GPS Block IIF satellites but it has not been observed on GPS L1 signals (EU-US, 2015). Furthermore, combination of signals on different frequencies assumes that the signals are synchronized in time at their broadcast. However, electronic components introduce different amounts of signal delay at 
different frequencies producing an inter-frequency bias that is nominally constant. This offset is estimated and broadcast to the user as part of the navigation data.

3.3. Differential code biases (DCBs)

In PPP, dual-frequency observations are typically used to eliminate the first order effects of the ionosphere. Hence, differences among the hardware dependent biases will appear in the observation equations, defined as differential code biases (DCBs). For example, when differencing the signals $c_{i}$ and $c_{j}$ on frequencies $i$ and $j$ :

$\mathrm{DCB}_{r_{G}}\left(c_{i}, c_{j}\right)=d_{r_{G}}\left(c_{i}\right)-d_{r_{G}}\left(c_{j}\right) ;$ and $\operatorname{DCB}^{k_{G}}\left(c_{i}, c_{j}\right)=d^{k_{G}}\left(c_{i}\right)-d^{k_{G}}\left(c_{j}\right)$

where $\mathrm{DCB}_{r_{G}}\left(c_{i}, c_{j}\right)$ and $\mathrm{DCB}^{k_{G}}\left(c_{i}, c_{j}\right)$ denote the receiver and satellite DCBs, respectively. When ionosphere-free combinations are applied, the DCBs for the frequencies $i$ and $j$ are scaled by the factors:

$$
\alpha_{i, j}=\frac{f_{i}^{2}}{f_{i}^{2}-f_{j}^{2}}, \quad \beta_{i, j}=\frac{f_{j}^{2}}{f_{i}^{2}-f_{j}^{2}}
$$

such that:

$\operatorname{DCB}_{r_{G}}\left(I F_{c_{i}, c_{j}}\right)=\alpha_{i, j} d_{r_{G}}\left(c_{i}\right)-\beta_{i, j} d_{r_{G}}\left(c_{j}\right) ;$ and $\operatorname{DCB}^{k_{G}}\left(I F_{c_{i}, c_{j}}\right)=\alpha_{i, j} d^{k_{G}}\left(c_{i}\right)-\beta_{i, j} d^{k_{G}}\left(c_{j}\right)$

where IF denotes the ionosphere-free operator. For example, the precise orbits and clock corrections provided by the IGS, are based on ionosphere-free combination of code and phase measurements. To minimize the biases problem, the IGS-MGEX lumps the associated DCBs into the receiver clock offsets. These "conventional" IGS clock corrections are based on $\mathrm{P}(\mathrm{Y})$ code measurements on L1/L2 frequencies for GPS, and E1/E5a for Galileo. Thus, assuming the primary signals are defined as $c_{1}$ and $c_{2}$, the satellite clock offset is (Montenbruck et al 2013):

$d t\left(I F_{c_{1}, c_{2}}\right)^{k_{G}}=d t^{k_{G}}-\operatorname{DCB}^{k_{G}}\left(I F_{c_{1}, c_{2}}\right)$ or $d t^{k_{G}}=d t\left(I F_{c_{1}, c_{2}}\right)^{k_{G}}+\operatorname{DCB}^{k_{G}}\left(I F_{c_{1}, c_{2}}\right)$

Hence, the ionosphere-free code observations modelled from these 'primary' frequencies will not include DCBs. However, for raw (undifferenced) observations and for ionosphere-free combination of other signals, DCBs should be included.

The ionosphere-free DCB ( $\mathrm{DCB}^{k_{G}}\left(I F_{c_{1}, c_{2}}\right)$ ) is not computed by IGS, and it can be formulated by premultiplying the $D C B^{k_{G}}$ in equation (3) by $\beta_{i, j}$ in one case and by $\alpha_{i, j}$ in another case, such that:

$$
\begin{array}{r}
\operatorname{DCB}^{k_{G}}\left(I F_{c_{1}, c_{2}}\right)=\alpha_{i, j} d^{k_{G}}\left(c_{1}\right)-\beta_{i, j} d^{k_{G}}\left(c_{2}\right) \\
=\beta_{1,2} D C B^{k_{G}}\left(c_{1}, c_{2}\right)+d^{k_{G}}\left(c_{1}\right) \\
=\alpha_{1,2} \operatorname{DCB}^{k_{G}}\left(c_{1}, c_{2}\right)+d^{k_{G}}\left(c_{2}\right)
\end{array}
$$

Thus, for the signal $c_{i}$, we have:

$$
d t^{k_{G}}+d^{k_{G}}\left(c_{i}\right)=d t\left(I F_{c_{1}, c_{2}}\right)^{k_{G}}+\beta_{1,2} D C B^{k_{G}}\left(c_{1}, c_{2}\right)-D C B^{k_{G}}\left(c_{i}, c_{1}\right)
$$

Similarly in the case for the receiver biases.

For the satellite DCBs (i. e. DCB ${ }^{k_{G}}$ ), when processing the legacy L1 or L2 P(Y) signals, a Time Group Delay (TGD) parameter is provided in the GPS navigation message, which represents a scaled version of the satellite-differential L1/L2 P(Y) code. In addition, inter-signal corrections (ISCs) will be provided in the new civil navigation (CNAV) data of the L2C and L5 signals as well as the CNAV-2 message data for the future L1C signal. The TGDs and ISCs are fully equivalent to the satellite DCB (Montenbruck et al 2014). For legacy signals, the DCBs for GPS and GLONASS satellites available from the Centre for Orbit Determination in Europe (CODE) (Schaer and Dach 2010) are applicable for the dual-frequency code measurements with the naming convention consistent with Receiver Independent Exchange Format (RINEX) 
version 2 (e.g. C1, C2, P1 and P2). With the modernization of GPS and advent of multi-constellation GNSS, DCBs that are consistent with RINEX version 3 tracking modes should be used. Such DCBs for various code measurements and tracking modes of GPS, Galileo and BeiDou observations, with daily repeatability of 0.05-0.3 ns, are provided by MGEX (see ftp://cddis.gsfc.nasa.gov/pub/gps/products/mgex/ dcb). DCBs for Quasi-Zenith Satellite System (QZSS) are not provided at present since more than one operational satellite is needed to establish a constellation mean. Fig. 1 shows as an example the DCBs obtained from MGEX for GPS PRN 1, Galileo PRN E11 and BeiDou PRN C01 from January to July 2014. In general, the DCBs of various signals were between $-10.89 \mathrm{~ns}$ and $15.38 \mathrm{~ns}(-3.267 \mathrm{~m}$ and $4.614 \mathrm{~m})$, with an overall standard deviation of $0.52 \mathrm{~ns}$. During some period, the computed DCBs vary from their expected long-term stable values as shown in figure 1 . This variation can be attributed to a variation in the constellation mean. A possible method for validation of the DCBs is to treat them as quasi-observations, and apply the singlereceiver single-channel validation method described in El-Mowafy (2014a-c).

In PPP, users need to use appropriate DCBs to make code measurements consistent with the precise orbits and satellite clock corrections used. For example, since the IGS products are produced using the ionospherefree combination of $\mathrm{P}(\mathrm{Y})$ code measurements of GPS L1 and L2, dual-frequency PPP users measuring the $\mathrm{C} 1$ (C/A) code need to apply the P1-C1 DCB corrections to convert it to P1.

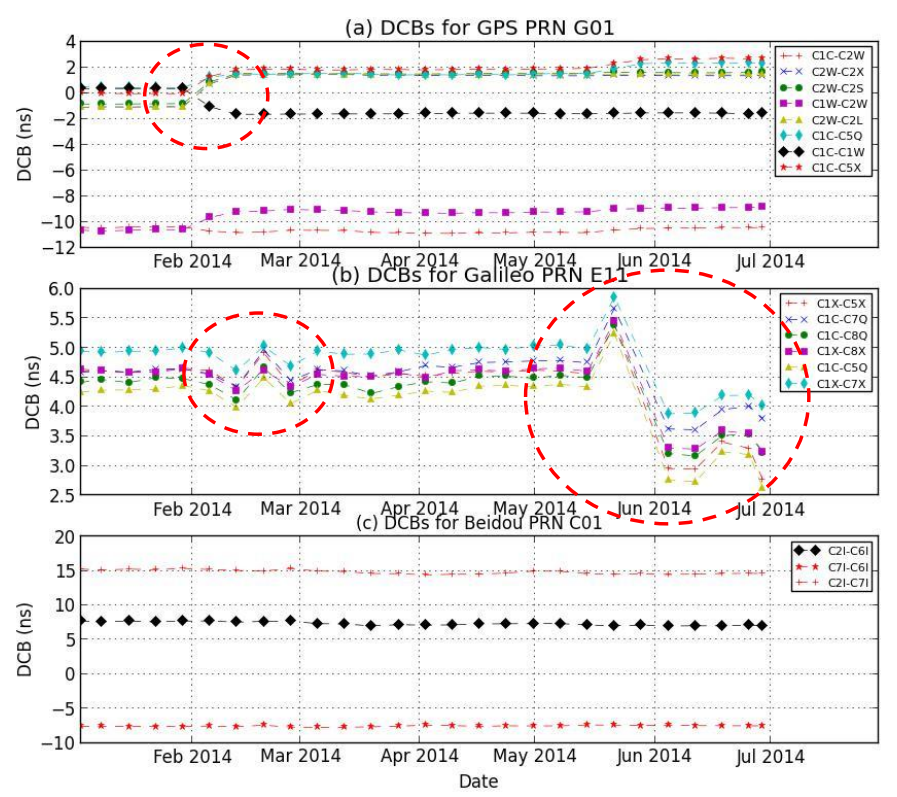

Figure 1. DCBs for (a) GPS PRN 01; (b) Galileo PRN E11; and (c) BeiDou PRN C01 from January to July 2014, obtained from IGS MGEX.

\subsection{Differential phase biases (DPBs)}

Similar to DCBs, the use of dual-frequency observations in PPP will result in Differential Phase Biases (DPBs), which are the difference between hardware dependent phase biases in the satellite and receiver. With reference to equation (2), and for signals $\left(c_{i}, c_{j}\right)$, the DPB in length units are:

$$
\begin{aligned}
& \operatorname{DPB}_{r_{G}}\left(c_{i}, c_{j}\right)=c\left\{\delta_{r_{G}}\left(c_{i}\right)-\delta_{r_{G}}\left(c_{j}\right)\right\} \\
& \operatorname{DPB}^{k_{G}}\left(c_{i}, c_{j}\right)=c\left\{\delta^{k_{G}}\left(c_{i}\right)-\delta^{k_{G}}\left(c_{j}\right)\right\}
\end{aligned}
$$

and for the ionosphere-free case we have:

$$
\begin{aligned}
& \operatorname{DPB}_{r_{G}}\left(I F_{c_{i}, c_{j}}\right)=\alpha_{i, j} \delta_{r_{G}}\left(c_{i}\right)-\beta_{i, j} \delta_{r_{G}}\left(c_{j}\right) \\
& \operatorname{DPB}^{k_{G}}\left(I F_{c_{i}, c_{j}}\right)=\alpha_{i, j} \delta^{k_{G}}\left(c_{i}\right)-\beta_{i, j} \delta^{k_{G}}\left(c_{j}\right)
\end{aligned}
$$


The satellite and receiver DPBs and IFPBs are difficult to be estimated independently since they are almost constant and as they have the same characteristics of phase ambiguities, they are practically inseparable. Hence, prior-accounting for the satellite DPBs and IFPB is crucial for multi-frequency PPP with integerambiguity solution.

3.5. Inter-system biases (ISBS)

Assuming that the signal characteristics of the satellites from the same GNSS constellation on the same spectral occupation are identical, it is generally assumed that the receiver-dependent biases are the same for each satellite. However, for multi-constellation data, it cannot be assumed that receiver hardware biases are the same for signals from different GNSS constellations, even if they are transmitted on the same frequency (Hegarty et al 2004). Hence, multi-constellation PPP models must deal with the inter-system biases (ISBs).

To enable joint processing of measurements from different systems, all observations should refer to a reference system time scale and when using observations from other systems add the difference between their system clock offset and the reference system clock offset. At the satellite end this is dealt with as the $I S T B$. However, at the receiver end, the inter-system clock offset (denoted as ISB) is receiver-specific and has to be estimated as an additional parameter for each system used in conjunction with GPS. For example, for Galileo, assuming that the primary frequencies for Galileo are denoted as $\left(E_{1}, E_{5 a}\right)$, the $I S B$ for ionosphere-free combinations is (Montenbruck et al 2013):

$I S B_{E-G}=d t\left(I F_{E_{1}, E_{5 a}}\right)_{r_{E}}-d t\left(I F_{c_{1}, c_{2}}\right)_{r_{G}}$

and for the individual uncombined observations it is:

$$
I S B_{E-G}=d t_{r_{E}}-d t_{r_{G}}
$$

Both ISTB and ISB can be combined as one parameter per constellation that is additional to GPS. Odijk et al. (2013) showed that ISBs differ for receivers from different manufacturers and that they are stable over several hours. The magnitude of these ISBs reached up to $0.13 \mathrm{~ns}$ and $5.5 \mathrm{~ns}$ for phase and code observations, respectively. ISBs can also be absorbed into the estimated receiver clock offset such that a different receiver clock offset is associated with each system.

\section{Biases in the PPP models}

This section describes parametrization of biases in single- and multi-constellation PPP. Two cases will be discussed; the ionosphere-free dual-frequency combination of primary signals; and ionosphere-free combination involving measurements other than the primary ones. The use of between-satellite single difference (BSSD) observation model and MGEX or similar products are utilized to account for some biases.

\subsection{Ionosphere-free dual-frequency combination of primary signals}

As discussed earlier, using IGS products, the ionosphere-free code observations modelled from the 'primary' frequencies (defined as $c_{1}$ and $c_{2}$ ) will not include satellite DCBs, i. e. DCB ${ }_{G}\left(I F_{c_{1}, c_{2}}\right)$, such that the code observation equation after applying corrections for the satellite clock offset $\left(d t\left(I F_{c 1, c 2}\right)^{k_{G}}\right)$ is:

$$
P\left(I F_{c_{1}, c_{2}}\right)_{r}^{k_{G}}=\rho_{r}^{k_{G}}+c d t\left(I F_{c_{1}, c_{2}}\right)_{r_{G}}+D C B_{r_{G}}\left(I F_{c_{1}, c_{2}}\right)+T^{k_{G}}+\varepsilon_{P\left(I F_{c_{1}, c_{2}}\right)_{r}^{k_{G}}}
$$

Given that the ionosphere-free satellite clock corrections comprises clock offsets and DCBs, using the same clock corrections in ionosphere-free phase observation equations will bring in satellite DCBs, where:

$$
\begin{gathered}
\phi\left(I F_{c_{1}, c_{2}}\right)_{r}^{k_{G}}=\rho_{r}^{k_{G}}+c d t\left(I F_{c_{1}, c_{2}}\right)_{r_{G}}+c \operatorname{DCB}^{k_{G}}\left(I F_{c_{1}, c_{2}}\right)+D P B_{r_{G}}\left(I F_{c_{1}, c_{2}}\right)-D P B^{k_{G}}\left(I F_{c_{1}, c_{2}}\right)+ \\
\operatorname{IFPB}\left(I F_{c_{1}, c_{2}}\right)+T^{k_{G}}+\lambda_{I F_{c_{1}, c_{2}}} N_{r}^{k_{G}}\left(I F_{c_{1}, c_{2}}\right)+\varepsilon_{\phi\left(I F_{c_{1}, c_{2}}\right)_{r}^{k_{G}}}
\end{gathered}
$$

which can be re-written as: 


$$
\begin{aligned}
\phi\left(I F_{c_{1}, c_{2}}\right)_{r}^{k_{G}}= & \rho_{r}^{k_{G}}+c d t\left(I F_{c_{1}, c_{2}}\right)_{r_{G}}+v\left(I F_{c_{1}, c_{2}}\right)_{r_{G}}-v\left(I F_{c_{1}, c_{2}}\right)^{k_{G}}+T^{k_{G}}+\lambda_{I F_{c_{1}, c_{2}}} N_{r}^{k_{G}}\left(I F_{c_{1}, c_{2}}\right)+ \\
& \varepsilon_{\phi\left(I F_{c_{1}, c_{2}}\right)_{r}^{k_{G}}}
\end{aligned}
$$

where $v\left(I F_{c_{1}, c_{2}}\right)_{r_{G}}$ and $v\left(I F_{c_{1}, c_{2}}\right)^{k_{G}}$ are lumped receiver and satellite nuisance bias terms, such that:

$$
\begin{aligned}
& v\left(I F_{c_{1}, c_{2}}\right)^{k_{G}}=c \operatorname{DCB}^{k_{G}}\left(I F_{c_{1}, c_{2}}\right)+\operatorname{DPB}^{k_{G}}\left(I F_{c_{1}, c_{2}}\right) \\
& v\left(I F_{c_{1}, c_{2}}\right)_{r_{G}}=\operatorname{DPB}_{r_{G}}\left(I_{c_{1}, c_{2}}\right)+\operatorname{IFPB}\left(I F_{c_{1}, c_{2}}\right)
\end{aligned}
$$

where $\operatorname{IFPB}\left(\operatorname{IF}_{c_{1}, c_{2}}\right)=\alpha_{1,2} \operatorname{IFPB}\left(c_{1}\right)-\beta_{1,2} \operatorname{IFPB}\left(c_{2}\right)$. Note that $D C B^{k_{G}}\left(I_{c_{1}, c_{2}}\right)$ is not estimated by MGEX. Although $D P B$ and $I F P B$ are not estimated separately and hence considered in most of the literature as one term, we leave them separate without being estimated independently in the formulation since in principle they have assumed different characteristics as explained in section 2.

For parameterization in a solution model, receiver biases are hardware-dependent and thus require either to be individually calibrated or be estimated as additional unknowns. Instead, under the assumption that the receiver code hardware biases from the same spectral occupation of a GNSS constellation are the same for all satellites from the same constellation, using BSSD model will cancel $d t\left(I F_{c_{1}, c_{2}}\right)_{r_{G}}, D C B_{r_{G}}\left(I F_{c_{1}, c_{2}}\right)$ and $v\left(I F_{c_{1}, c_{2}}\right)_{r_{G}}$. The main challenge left is to separate $v\left(I F_{c_{1}, c_{2}}\right)^{k_{G}}$ from the phase ambiguities per satellite since they have the same modelling and characteristics and solving for the two would introduce rank deficiency. Since $v\left(I F_{c_{1}, c_{2}}\right)^{k_{G}}$ is satellite dependent, and due to the fact that these biases are usually stable during a typical observation session of a few hours; they can be estimated using a network of known stations and be applied by the user. This can either be for the whole quantity or only for the fractional part (also known as fractional cycle bias - FCB), where for the latter case the integer part can be added to the individual integer ambiguities that can be solved using known methods, such as the least-squares ambiguity decorrelation adjustment method (LAMBDA). One problem here is the short wave length of the ionosphere-free combination for some combinations. For example, for L1/L2 ionosphere combination the wavelength is 6.3 $\mathrm{mm}$, computed as $\frac{2 \times c \times f_{0}}{f_{1}^{2}-f_{2}^{2}}$, for integer ambiguity combination $\left(77 N_{l}-60 N_{2}\right)$, where $f_{o}$ is $10.23 \mathrm{MHz}$. Hence, the ambiguities cannot be solved directly.

Several methods have been presented to solve the ionosphere-free PPP ambiguities. For example, under the assumption that a service provider supplies the wide-lane and narrow-lane phase biases, denoted as $v\left(w l_{c_{1}, c_{2}}\right)^{k_{G}}$ and $v\left(n l_{c_{1}, c_{2}}\right)^{k_{G}}$ respectively, which are estimated from an ambiguity-fixed network solution (Ge et al 2008). The wide-lane biases are stable over several hours to a few days, whereas the narrow-lane biases are only stable for a shorter period (15 minutes to two hours); therefore, they can be updated with different rates. At the user end, we solve first for the wide-lane ambiguities using code and carrier Melbourne-Wübbena linear combination with the received values for $v\left(w l_{c_{1}, c_{2}}\right)^{k_{G}}$. Next, narrow-lane ambiguities are formed using the ionosphere-free observations and their resolution is attempted (for more details see Ge et al 2008 and Geng et al 2012). Once the wide-lane and narrow-lane integer ambiguities are fixed, the ionosphere-free ambiguities are computed as (Geng et al 2012):

$\lambda_{I F_{c_{1}, c_{2}}} \widetilde{N}_{r}^{k_{G}}\left(I F_{c_{1}, c_{2}}\right)=\frac{f_{1}}{f_{1}+f_{2}} \lambda_{n l_{c_{1}, c_{2}}}\left\{N_{r}^{k_{G}}(n l)+v\left(n l_{c_{1}, c_{2}}\right)^{k_{G}}\right\}+\frac{f_{1} f_{2}}{f_{1}^{2}-f_{2}^{2}} \lambda_{w l_{c_{1}, c_{2}}} N_{r}^{k_{G}}(w l)$

where $\lambda_{I F_{c_{1}, c_{2}}} \widetilde{N}_{r}^{k_{G}}\left(I F_{c_{1}, c_{2}}\right)$ replaces $\left(\lambda_{I F_{c_{1}, c_{2}}} N_{r}^{k_{G}}\left(I F_{c_{1}, c_{2}}\right)+v\left(I F_{c_{1}, c_{2}}\right)^{k_{G}}\right)$ in equation (17), $N_{r}^{k_{G}}(n l)$ and $N_{r}^{k_{G}}(w l)$ are the narrow-lane and wide-lane integer ambiguities, respectively, $\lambda_{n l_{c_{1}, c_{2}}}$ and $\lambda_{w l_{c_{1}, c_{2}}}$ are their corresponding wavelengths.

Similarly for other constellations, for example Galileo, using the primary frequencies (assumed as $E_{l}, E_{5 a}$ ) for satellite $l$ we have: 
$P\left(I F_{E_{1}, E_{5 a}}\right)_{r}^{l_{E}}=\rho_{r}^{l_{E}}+c\left\{d t\left(I F_{c_{1}, c_{2}}\right)_{r_{G}}+I S B_{E-G}+D C B_{r_{E}}\left(I F_{E_{1}, E_{5 a}}\right)-I S T B_{E-G}\right\}+T^{l_{E}}+\varepsilon_{P\left(I F_{E_{1}, E_{5 a}}\right)_{r}^{l_{E}}}$

where the satellite clock offset $d t\left(I F_{E_{1}, E_{5} a}\right)^{l_{E}}$ is eliminated by the use of clock corrections. It is assumed that these clock corrections include the DCB component $\operatorname{DCB}^{l_{E}}\left(I F_{E_{1}, E_{5 a}}\right)$ similar to the case of GPS. The $I S T B_{E-G}$ can be eliminated in the future when using MGEX clock corrections as mentioned earlier. Similarly, the phase observation equation reads:

$$
\begin{aligned}
\phi\left(I F_{E_{1}, E_{5 a}}\right)_{r}^{l_{E}}= & \rho_{r}^{l_{E}}+c\left\{d t\left(I F_{c_{1}, c_{2}}\right)_{r_{G}}+I S B_{E-G}-I S T B_{E-G}\right\}+T^{l_{E}}+v\left(I F_{E_{1}, E_{5 a}}\right)_{r_{E}}-v\left(I F_{E_{1}, E_{5 a}}\right)^{l_{E}}+ \\
& \lambda_{I F_{E_{1}, E_{2}}} N_{r}^{l_{E}}\left(I F_{E_{1}, E_{5 a}}\right)+\varepsilon_{\phi\left(I F_{E_{1}, E_{5} a}\right)_{r}^{l_{E}}}
\end{aligned}
$$

with

$$
\begin{aligned}
& v\left(I F_{E_{1}, E_{5} a}\right)^{l_{E}}=c \operatorname{DCB}^{l_{E}}\left(I F_{E_{1}, E_{5} a}\right)+\operatorname{DPB}^{l_{E}}\left(I F_{E_{1}, E_{5} a}\right) \\
& v\left(I F_{E_{1}, E_{5}}\right)_{r_{E}}=\operatorname{DPB}_{r_{E}}\left(I_{E_{1}, E_{5} a}\right)+\operatorname{IFPB}\left(I_{E_{1}, E_{5 a}}\right)
\end{aligned}
$$

When referencing to a pivot GPS satellite, for example, the use of the BSSD model will eliminate $d t\left(I F_{c_{1}, c_{2}}\right)_{r_{G}}$; however, it cannot remove $I S B_{E-G}$, which is a receiver time offset between GPS and Galileo.

In summary, the terms $I S B_{E-G}, I S T B_{E-G}, D C B_{r_{E}}\left(I F_{E_{1}, E_{5} a}\right)$ and $v\left(I F_{E_{1}, E_{5 a}}\right)_{r}$ are assumed common for all Galileo satellites and need to be estimated. To reduce number of unknowns, ISBE-G ISTB $B_{E-G}$ and $D C B_{r_{E}}\left(I F_{E_{1}, E_{5} a}\right)$ are lumped into one term for code observations (they are inseparable anyway as they share the same parameterization in the model). Similarly, $I S B_{E-G}, I S T B_{E-G}$ and $v\left(I F_{E_{1}, E_{5} a}\right)_{r_{E}}$ are lumped into one term for phase observations. In the same way, two terms are introduced when integrating additional constellations with GPS.

\subsection{Ionosphere-free dual-frequency combination of signals that involve measurements other than the primary ones}

The models presented so far can be extended to include ionosphere-free combinations using observations of a third or fourth frequency (e.g. L5 for GPS, E5b or E6 for Galileo) or when using signals from different tracking modes (i.e. different signals modulated on the same frequency). Let us consider the general case using signals $c_{i}$ and $c_{j}$ that are different from the primary signals $\mathrm{c}_{1}$ and $\mathrm{c}_{2}$ from constellation $G$, the ionosphere-free code observation equation is:

$$
\begin{aligned}
P\left(I F_{c_{i}, c_{j}}\right)_{r}^{k_{G}} & =\rho_{r}^{k_{G}}+c\left\{d t\left(I F_{c_{1}, c_{2}}\right)_{r_{G}}+D C B_{r_{G}}\left(I F_{c_{i}, c_{j}}\right)-\left(D C B^{k_{G}}\left(I F_{c_{i}, c_{j}}\right)-D C B^{k_{G}}\left(I F_{c_{1}, c_{2}}\right)\right)\right\}+T^{k_{G}}+ \\
& \varepsilon_{P\left(I F_{c_{i}, c_{j}}\right)_{r}^{k_{G}}}
\end{aligned}
$$

where the satellite clock offset $d t\left(I F_{c 1, c 2}\right)^{k_{G}}$ is eliminated by the use of its correction, and $D C B^{k_{G}}\left(I F_{c_{1}, c_{2}}\right)$ re-appeared due to the use of clock corrections that include the bias of the primary frequencies. The bias term $\operatorname{DCB}^{k_{G}}\left(I F_{c_{i}, c_{j}}\right)$ reads (Montenbruck et al 2013):

$\operatorname{DCB}^{k_{G}}\left(I F_{c_{i}, c_{j}}\right)=\alpha_{i, j} D C B^{k_{G}}\left(c_{i}, I F_{c_{1}, c_{2}}\right)-\beta_{i, j} D C B^{k_{G}}\left(c_{j}, I F_{c_{1}, c_{2}}\right)$

where:

$\operatorname{DCB}^{k_{G}}\left(c_{i}, I F_{c_{1}, c_{2}}\right)=\left\{-\beta_{1,2} D C B^{k_{G}}\left(c_{1}, c_{2}\right)+\operatorname{DCB}^{k_{G}}\left(c_{i}, c_{1}\right)\right\}$ 
and similarly $\operatorname{DCB}^{k_{G}}\left(c_{j}, I F_{c_{1}, c_{2}}\right)$. An expression for $\operatorname{DCB}_{r_{G}}\left(I F_{c_{i}, c_{j}}\right)$ can be obtained in the same way as $\operatorname{DCB}^{k_{G}}\left(I F_{c_{i}, c_{j}}\right)$.

In principle, without biases, $d t\left(I F_{c_{1}, c_{2}}\right)_{r_{G}}$ should be equivalent to $d t\left(I F_{c_{i}, c_{j}}\right)_{r_{G}}$ and $d t\left(I F_{c 1, c 2}\right)^{k_{G}}$ should be

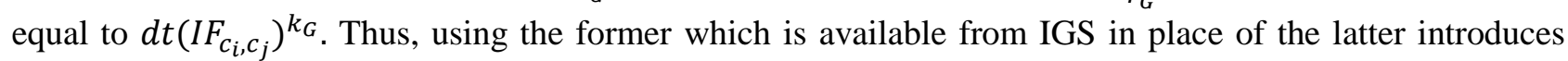
$\operatorname{DCB}^{k_{G}}\left(I F_{c_{1}, c_{2}}\right)$ in the phase equation, such that:

$$
\begin{aligned}
\phi\left(I F_{c_{i}, c_{j}}\right)_{r}^{k_{G}}= & \rho_{r}^{k_{G}}+c d t\left(I F_{c_{1}, c_{2}}\right)_{r_{G}}+v\left(I F_{c_{i}, c_{j}}\right)_{r}-v\left(I F_{c_{i}, c_{j}}\right)^{k_{G}}+T^{k_{G}}+\lambda_{I F_{c_{i}, c_{j}}} N_{r}^{k_{G}}\left(I F_{c_{i}, c_{j}}\right)+ \\
& \varepsilon_{\phi\left(I F_{c_{i}, c_{j}}\right)_{r}^{k_{G}}}
\end{aligned}
$$

where

$$
\begin{aligned}
& v\left(I F_{c_{i}, c_{j}}\right)^{k_{G}}=c \operatorname{DCB}^{k_{G}}\left(I F_{c_{1}, c_{2}}\right)+\operatorname{DPB}^{k_{G}}\left(I F_{c_{i}, c_{j}}\right) \\
& v\left(I F_{c_{i}, c_{j}}\right)_{r_{G}}=\operatorname{DPB}_{r_{G}}\left(I F_{c_{i}, c_{j}}\right)+\operatorname{IFPB}\left(I F_{c_{i}, c_{j}}\right)
\end{aligned}
$$

Again, the receiver offsets $D C B_{r_{G}}\left(I F_{c_{i}, c_{j}}\right)$ and $d t\left(I F_{c_{1}, c_{2}}\right)_{r_{G}}$ are assumed the same for all satellites from the same spectral occupation of a GNSS constellation. Therefore, they will cancel when applying BSSD model. For constellations other than GPS, for example Galileo, and using signals $E_{i}$ and $E_{j}$ that are different from the primary signals $\left(E_{1}, E_{5 a}\right)$ used in the generation of the clock corrections, the equation of ionosphere-free combination for code observations is:

$$
\begin{aligned}
P\left(I F_{E_{i}, E_{j}}\right)_{r}^{l_{E}}= & \rho_{r}^{l_{E}}+c\left\{d t\left(I F_{c_{1}, c_{2}}\right)_{r_{G}}+I S B_{E-G}-I S T B_{E-G}+\operatorname{DCB}_{r_{E}}\left(I F_{E_{i}, E_{j}}\right)-\left(D C B^{l_{E}}\left(I F_{E_{i}, E_{j}}\right)-\right.\right. \\
& \left.\left.D C B^{l_{E}}\left(I F_{E_{1}, E_{5 a}}\right)\right)\right\}+T^{l_{E}}+\varepsilon_{P\left(I F_{E_{i}, E_{j}}\right)_{r}}^{l_{E}}
\end{aligned}
$$

where $D C B_{r_{E}}\left(I F_{E_{i}, E_{j}}\right), D C B^{l_{E}}\left(I F_{E_{i}, E_{j}}\right), \alpha_{i, j}$ and $\beta_{i, j}$ are formulated using equations $(4,5$ and 7$)$ employing Galileo frequencies, and $D C B^{l_{E}}\left(I F_{E_{1}, E_{5}}\right)$ re-appears due to use of clock corrections that include this bias. However, as mentioned earlier, the use of a pivot GPS satellite will not eliminate the $I S B_{E-G}, I S T B_{E-G}$ and these DCBs; therefore, they need to be estimated. In analogy with equation (22), the phase observation equation in this case will be:

$$
\begin{aligned}
\phi\left(I F_{E_{i}, E_{j}}\right)_{r}^{l_{E}=} & \rho_{r}^{l_{E}}+c\left\{d t\left(I F_{c_{1}, c_{2}}\right)_{r_{G}}+I S B_{E-G}-I S T B_{E-G}\right\}+T^{k_{G}}+v\left(I F_{E_{i}, E_{j}}\right)_{r_{E}}-v\left(I F_{E_{i}, E_{j}}\right)^{l_{E}}+ \\
& \lambda_{I F_{E_{i}, E_{j}}} N_{r}^{l_{E}}\left(I F_{E_{i}, E_{j}}\right)+\varepsilon_{\phi\left(I F_{E_{i}, E_{j}}\right)_{r}^{l_{E}}}
\end{aligned}
$$

where

$$
\begin{aligned}
& v\left(I F_{E_{i}, E_{j}}\right)^{l_{E}}=c \mathrm{DCB}^{l_{E}}\left(I F_{c_{1}, c_{2}}\right)+\operatorname{DPB}^{l_{E}}\left(I F_{E_{i}, E_{j}}\right) \\
& v\left(I F_{E_{i}, E_{j}}\right)_{r_{E}}=\operatorname{DPB}_{r_{E}}\left(I F_{E_{i}, E_{j}}\right)+\operatorname{IFPB}\left(I F_{E_{i}, E_{j}}\right)
\end{aligned}
$$

The case where either one of the signals $i$ or $j$ is one of the primary signals (i.e. $i=1$ or 2 , or $j=1$ or 2 ) can easily be derived by replacing $i$ or $j$ by 1 or 2 in Eqs. $25-33$. As discussed earlier, $I S B_{E-G,} I S T B_{E-G}$ and $D C B_{r_{E}}\left(I F_{E_{i}, E_{j}}\right)$ are lumped into one term in code observations and likewise $I S B_{E-G,}, I S T B_{E-G}$ and $v\left(I F_{E_{i}, E_{j}}\right)_{r}$ are joined in a second term for phase observations. Both terms are common for all Galileo satellite observations on frequencies $i$ and $j$. 
Table 1 gives some options discussed so far for the treatment of a number of biases in single-constellation and multi-constellation PPP processing. In summary, products from major service providers such as IGS, MGEX and CODE are designed to minimize the impact of DCBs when ionosphere-free primary signals are used. However, the use of other types of observations will include DCBs that need to be accounted for during processing. In addition, when integrating observations from multiple constellations, several biases remain and thus need to be estimated such as ISBs which are receiver dependent. A user should carefully consider the interoperability of the externally provided biases by utilizing consistent modelling of these biases within the observation equations with that are compatible with the ones used at the service network to generate these biases. This will need also some details that have not been addressed in this manuscript, such as the use of the same satellite attitude convention in order to guarantee a consistent computation of the phase wind-up.

Table 1. Summary of possible treatment of biases in multi-constellation multi-frequency PPP.

\begin{tabular}{|c|c|c|}
\hline Bias type & Method of treatment & Remarks \\
\hline $\begin{array}{l}\text { Satellite hardware bias } \\
\text { (if undifferenced frequencies are } \\
\text { considered; i.e. no DCB or DPB) }\end{array}$ & $\begin{array}{l}\text { Code biases are removed using BSSD } \\
\text { model; phase biases are absorbed in } \\
\text { phase ambiguities if untreated (resulting } \\
\text { in float ambiguities). For PPP with } \\
\text { ambiguity fixing, they are combined with } \\
\text { IFPB and externally obtained. }\end{array}$ & $\begin{array}{l}\text { Satellite phase hardware biases are } \\
\text { relatively stable during operation of } \\
\text { a few hours. }\end{array}$ \\
\hline Satellite DCBs & $\begin{array}{l}\text { Use IGS MGEX DCB products when } \\
\text { needed. }\end{array}$ & $\begin{array}{l}\text { Not present for the primary code } \\
\text { observations GPS L1/L2 P(Y), and } \\
\text { Galileo E1/E5a but affects phase } \\
\text { observations and are present in } \\
\text { other types of observations. }\end{array}$ \\
\hline Satellite DPBs & $\begin{array}{l}\text { Calibrated by external information } \\
\text { (within the term } v \text { ); or considered to be } \\
\text { absorbed in the phase ambiguities (i.e. } \\
\text { float ambiguities). }\end{array}$ & $\begin{array}{l}\text { Satellite DPBs result in non-integer } \\
\text { ambiguity terms. }\end{array}$ \\
\hline $\begin{array}{l}\text { Receiver hardware biases } \\
\text { (if undifferenced frequencies are } \\
\text { considered; i.e. no DCB or DPB) }\end{array}$ & $\begin{array}{l}\text { Assumed cancelled for the same } \\
\text { frequency when using BSSD model. }\end{array}$ & \\
\hline Receiver DCBs & $\begin{array}{l}\text { Assumed cancelled when using BSSD } \\
\text { model for the same frequencies for a } \\
\text { single constellation, but needs to be } \\
\text { estimated in case of multiple GNSS with } \\
\text { a pivot GPS satellite. }\end{array}$ & \\
\hline Receiver DPBs & $\begin{array}{l}\text { Assumed cancelled when using BSSD } \\
\text { for the same frequencies for a single } \\
\text { constellation; can be absorbed with } \\
\text { ambiguities in case of multiple GNSS. }\end{array}$ & \\
\hline $\begin{array}{l}\text { ISTB among satellite clock } \\
\text { corrections of multi-constellations }\end{array}$ & $\begin{array}{l}\text { Needs to be estimated, combined with } \\
\text { receiver ISB. }\end{array}$ & $\begin{array}{l}\text { Future MGEX clock corrections for } \\
\text { all constellations will be referenced } \\
\text { to GPS. }\end{array}$ \\
\hline ISBs & $\begin{array}{l}\text { ISB at the receiver should be estimated } \\
\text { as a parameter, can be combined with } \\
\text { ISTB and receiver DCB. }\end{array}$ & $\begin{array}{l}\text { ISB is individual receiver- } \\
\text { dependent parameter per each } \\
\text { additional constellation to GPS. }\end{array}$ \\
\hline
\end{tabular}

\section{Testing}

Firstly, the impact of taking biases such as DCBs into account is demonstrated using simulated data with an epoch interval of 30 seconds at the IGS station CEDU in Australia. L1, L2 and L5 GPS signals were simulated for all satellites. The PPP algorithm was implemented as discussed above for three cases. The first is when using only L1-L2 ionosphere-free combination. The second case is when using L1-L5 ionospherefree combination, and finally when combining the two combinations, defined here as L1-L2-L5. The analysis was carried out firstly by applying the satellite DCBs that are obtained from MGEX in a float PPP solution, and secondly without applying them. The BSSD model was used in all cases. Analysis of the PPP results for 
the first 1000 epochs of data $(8 \mathrm{~h}: 20 \mathrm{~m})$ are presented in Table 2. The results are compared in terms of positioning convergence time, accuracy and precision. The convergence time is defined as the time when a 3 -dimentional positional precision of $0.05 \mathrm{~m}$ is reached and maintained. Accuracy is described by the mean of the errors (defined as the difference between computed and known station coordinates) after convergence is achieved, and precision is presented by the solution standard deviations (Stdev).

Table 2. Results for GPS L1-L2, L1-L5 and triple-frequency PPP with/without DCBs.

\begin{tabular}{lllllllll}
\hline Algorithm & $\begin{array}{l}\text { DCB } \\
\text { applied } \\
\text { Yes / No }\end{array}$ & $\begin{array}{l}\text { Conver- } \\
\text { gence } \\
\text { time } \\
(\mathrm{min})\end{array}$ & $\begin{array}{l}\text { Easting } \\
\text { Mean } \\
\text { Err } \\
(\mathrm{mm})\end{array}$ & $\begin{array}{l}\text { Northing } \\
\text { Mean } \\
\text { Err } \\
(\mathrm{mm})\end{array}$ & $\begin{array}{l}\text { Up } \\
\text { Mean } \\
\text { Err } \\
(\mathrm{mm})\end{array}$ & $\begin{array}{l}\text { Easting } \\
\text { Stdev } \\
(\mathrm{mm})\end{array}$ & $\begin{array}{l}\text { Northing } \\
\text { Stdev } \\
(\mathrm{mm})\end{array}$ & $\begin{array}{l}\text { Up } \\
\text { Stdev } \\
(\mathrm{mm})\end{array}$ \\
\hline L1-L2 IF & $\begin{array}{l}\text { Not } \\
\text { needed }\end{array}$ & 19 & 2 & 1 & 1 & 3 & 2 & 6 \\
\hline L1-L5 IF & $\mathrm{Y}$ & 19 & 1 & 0 & -2 & 1 & 1 & 4 \\
\hline L1-L2-L5 & $\mathrm{N}$ & 27.5 & 6 & -1 & -1 & 6 & 6 & 6 \\
\hline & $\mathrm{N}$ & 19 & -1 & 0 & -1 & 3 & 1 & 5 \\
\hline
\end{tabular}

The L1-L2 IF are the primary signals used to generate the precise orbits and clock products, and thus the DCBs were removed in code observations and absorbed with the float ambiguities in phase observations. For the L1-L5 IF case, the solution when the DCBs were applied converged earlier by 8.5 minutes from the solution without using DCBs and the standard deviations were significantly better. The L1-L5 IF results with DCB corrections were slightly better than the L1-L2 IF results. This is because the noise frequencydependent propagation factor for the L1-L5 IF combination (2.588), propagated from the noise of the unreferenced signals, is less than the L1-L2 IF factor (2.978). It is clear that any combination that involves L5 (i.e. L1-L5 and L1-L2-L5) gave poorer results when DCBs were not included.

Next, testing was performed in 14 January 2015 in the static mode using real data with 30 seconds observation intervals of the IGS station DLF1 and using IGS rapid precise orbits and clock corrections. The phase biases were computed from 12 IGS stations, located in the Netherlands, Belgium, Germany and France. The BSSD model was used. The data spanned approximately 75 minutes where only GPS observations were used at this stage. We plan to include other constellations in our future work. During the test period, 10 GPS satellites were observed, only 4 of which were from Block IIF with L5 frequency; hence, positioning was performed with a mix of dual and triple-frequency observations. The data was processed twice. In the first time, integer-ambiguity fixing was performed; and in the second processing time only float ambiguities were determined. The results of the two approaches were compared. The DCBs were used in both cases. Phase biases were estimated for all satellite pairs by averaging the fractional parts of all involved ambiguity estimates derived from the network solution. At the user end, the float $w l$ ambiguities were estimated using Melbourne-Wübbena observation combination and the $w l$ phase biases were employed to fix them to integers. This process was performed within a few seconds. The integer $w l$ ambiguities were next used as fixed parameters to estimate the $n l$ phase ambiguities. Once these $n l$ float ambiguities were corrected with the $n l$ phase biases, their integer values were resolved by applying the LAMBDA method. The obtained ambiguity fixing rate was $97 \%$. The ionosphere-free combinations with integer ambiguities were next formed to determine the position coordinates (as shown in Geng et al., 2012), receiver clock offset and troposphere zenith wet delay (ZTD) where the dry troposphere was modelled out using Saastamoinen model.

Figure 2 shows the horizontal and vertical solution standard deviations of the float-ambiguity solution and Figure 3 depicts results of processing the same data set but with integer ambiguity fixing. The horizontal stdev is the square root of the sum of the stdevs in Easting and Northing. As Figure 3 shows, PPP with integer ambiguity resolution was able to significantly shorten the solution convergence time using a mix of dual and triple-frequency data. In the float ambiguity mode, $42 \mathrm{~min}$ and 20 minutes convergence time were needed to reach a precision below $5 \mathrm{~cm}$ and $10 \mathrm{~cm}$, respectively. On the other hand, such a level of precision was achieved after about 3 minutes by fixing the ambiguities. Table 3 shows the solution conversion time in both the float and integer ambiguity solution modes in addition to the average precision after converging to < 
$5 \mathrm{~cm}$ in Easting, Northing and Up position components. There was also an improvement in precision by a few $\mathrm{mm}$ in case of the ambiguity-fixed solution compared with the float solution, in particular for the Easting component.

Table 3. Float and ambiguity-fixed PPP results.

\begin{tabular}{cccccc}
\hline $\begin{array}{c}\text { Ambiguity } \\
\text { solution }\end{array}$ & $\begin{array}{c}\text { Convergence } \\
\text { to } \\
<5 \mathrm{~cm}(\mathrm{~min})\end{array}$ & $\begin{array}{c}\text { Convergence } \\
\text { to } \\
<10 \mathrm{~cm}(\mathrm{~min})\end{array}$ & $\begin{array}{c}\text { Average } \\
\text { Easting stdev } \\
\text { converging to } \\
<5 \mathrm{~cm}(\mathrm{~cm})\end{array}$ & $\begin{array}{c}\text { Average } \\
\text { Northing stdev } \\
\text { converging to } \\
5 \mathrm{~cm}(\mathrm{~cm})\end{array}$ & $\begin{array}{c}\text { Average UP } \\
\text { stdev } \\
\text { converging to } \\
5 \mathrm{~cm}(\mathrm{~cm})\end{array}$ \\
\hline Float & 42 & 20 & 3.30 & 3.51 & 3.12 \\
\hline Integer & 3 & 3 & 2.29 & 3.02 & 3.04 \\
\hline
\end{tabular}
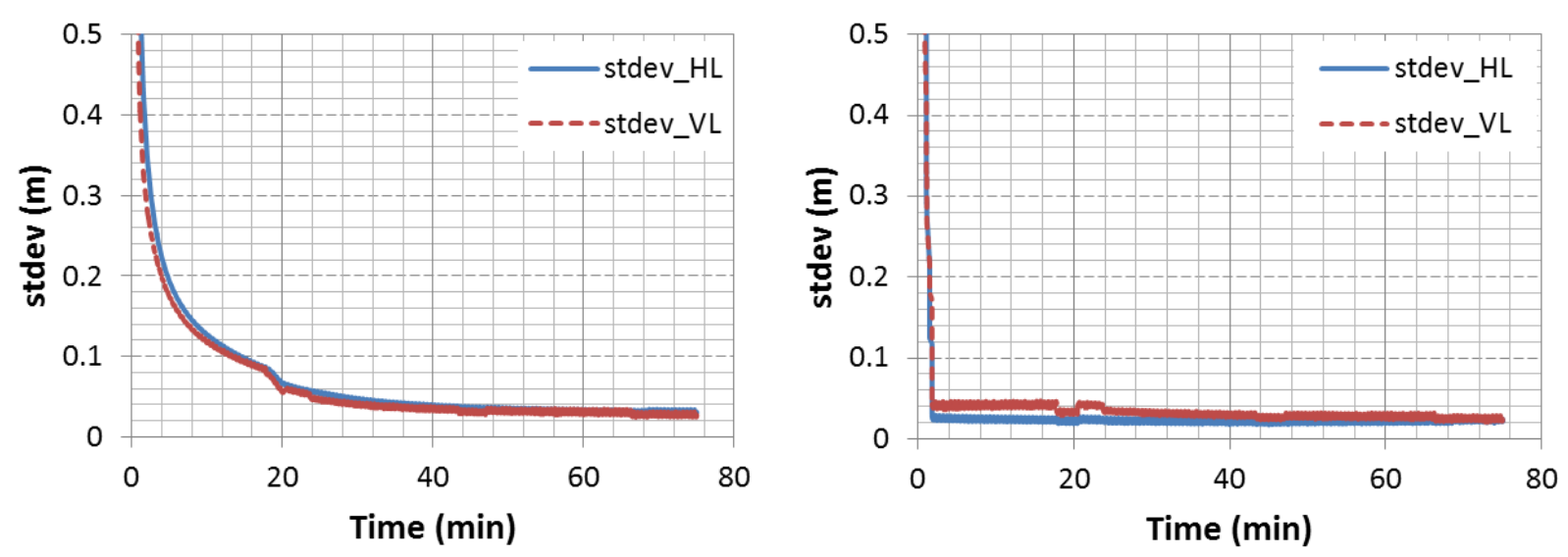

Figure 2. Positioning precision with float ambiguities. Figure 3. Positioning precision with fixed ambiguities. stdev_HL: horizontal stdev; stdev_VL: Vertical stdev.

\section{Conclusion}

Accounting for biases is necessary in order to reduce PPP convergence time and improve accuracy, and allow for integer ambiguity resolution. Single-constellation and multi-constellation PPP models were presented using ionosphere-free combination of measurements. Options for the treatment of different biases are summarized in Table 1. It is shown that biases that need to be considered in the observation equations vary according to the type and combination of signals used. We have two cases that have been presented in detail that allows for the use of all signals from multi-frequency GNSS. The first case is when using the ionosphere-free dual-frequency primary signals that are used for generation of clock corrections and precise orbits (such as L1 and L2 P(Y) for GPS, and E1 and E5a for Galileo). The second case is when using dualfrequency signals that are other than the primary ones.

The use of BSSD measurements from the same constellation is recommended as it cancels receiver-related biases including DCBs, receiver IFPB, receiver clock offset and common-mode satellite errors. However, these biases do not cancel when using differenced measurements between a pivot GPS satellite with satellites from other constellations. Additionally, ISBs and ISTB are introduced in this case. Currently, the use of MGEX products can compensate for satellite DCBs. IGS can play a major role in supporting multi-frequency and multi-constellation PPP users by producing precise orbits and clock corrections for each GNSS satellite and each individual frequency. This will enable PPP users to apply these corrections to raw measurements prior to forming any linear combination; thus, avoiding further complications. Furthermore, calibration of satellite (DPBs + IFPB) by an external organization would enable PPP users to perform integer ambiguity resolution resulting in significantly reduced solution convergence time. Results from the analysis of simulated data showed that it is necessary to apply corrections to DCB when using signals other than the primary ones. Results from tested GPS data set in a static mode showed that correcting for the biases allowed ambiguity-fixed PPP, which significantly shortened the solution convergence to 3 minutes at $<5 \mathrm{~cm}$ and 10 $\mathrm{cm}$ precision levels from almost $42 \mathrm{~min}$ and 20 minutes in the float ambiguity mode. An improvement in 
precision of a few mm was also achieved particularly in the Easting positioning component. Our future work will include application of the proposed models for the multi-constellation case.

\section{References}

Aggrey J, Bisnath S 2014 Analysis and modelling of pseudorange and carrier-phase biases in GNSS Precise Point Positioning, Proc. Of the $27^{\text {th }}$ International Technical Meeting of ION GNSS- 2014 Tampa FL Sept. 8-12 2014 2512-2522.

Banville, S., Santerre, R., Cocard, M. and R. Langley 2008 Satellite and Receiver Phase Bias Calibration for Undifferenced Ambiguity Resolution, Proceedings of the 2008 National Technical Meeting of The Institute of Navigation San Diego January 2008 711-719.

Cai C 2009 Precise Point Positioning Using Dual-Frequency GPS and GLONASS Measurements, Masters Thesis, Department of Geomatics Engineering University of Calgary Alberta Canada.

Collins, P Bisnath, S Lahaye, F and P Héroux 2010 Undifferenced GPS Ambiguity Resolution Using the Decoupled Clock Model and Ambiguity Datum Fixing, Navigation Journal of The Institute of Navigation 57(2) 123-135.

El-Mowafy A 2014a GNSS Multi-Frequency Receiver Single-Satellite Measurement Validation Method GPS Solutions 18(4) 553-561.

El-Mowafy A 2014b Estimation of Multi-Constellation GNSS Observation Stochastic Properties Using a Single-Receiver Single-Satellite Data Validation Method Survey Review 47-341 99-108.

El-Mowafy A 2014c Diagnostic Tools Using a Multi-Constellation Single-Receiver Single-Satellite Data Validation Method Journal of Navigation 68(1)196-214.

EU-U.S. Cooperation on Satellite Navigation Working Group 2015 C-ARAIM Technical Subgroup Milestone 2 Report http://www.gps.gov/policy/ cooperation/europe/2015/working-group-c/ARAIMmilestone-2-report.pd [Accessed 10 Nov 2015].

Ge M, Gendt G, Rothacher M, Shi C, Liu j 2008 Resolution of GPS Carrier Phase Ambiguities in Precise Point Positioning (PPP) with Daily Observations Journal of Geodesy 82 389-399.

Geng J, Shi C, Maorong Ge, Dodson A, Lou Y, Zhao Q, Liu j 2012 Improving the estimation of fractionalcycle biases for ambiguity resolution in precise point positioning Journal of Geodesy 86 579-589.

Hegarty C, Powers E, Fonville B 2004 Accounting for Timing Biases Between GPS, Modernized GPS, and Galileo Signals, 36th Annual Precise Time \& Time Interval (PTTI) Meeting Washington DC 7-9 Dec. 2004 307-317.

Khodabandeh A, Teunissen PJG 2014 Array-Based Satellite Phase Bias Sensing: Theory and GPS/BeiDou/QZSS Results, Meas. Sci. Technol. 25 (2014), 11pp.

Laurichesse D, Mercier F, Berthias JP, J Bijac 2008 Real-time Zero-Difference Ambiguities fixing and absolute RTK, Proc. ION NTM 2008 San Diego, Jan. 28-30 2008747 - 755.

Laurichesse D, Mercier F, Berthias JP, Brocca P, and Cerri, L. 2009 Integer ambiguity resolution on undifferenced GPS phase measurements and its application to $\mathrm{ppp}$ and satellite precise orbit determination Navigation 56(2) 135-149.

Laurichesse D 2015 Handling the Biases for Improved Triple-Frequency PPP Convergence, GPS World, 26(4), April 2015.

Li W, Teunissen PJG, Zhang B, Verhagen JS 2013 Precise Point Positioning Using GPS and Compass Observations, Sun et al. (eds.), China Satellite Navigation Conference (CSNC) 2013 Proceedings, Springer-Verlag Berlin Heidelberg, pp 367-378.

Montenbruck O, Hauschild A 2013 Code Biases in Multi-GNSS Point Positioning, Proc. ION-ITM-2013, 2830 Jan. 2013, San Diego, 616-628.

Montenbruck O, Hauschild A, Steigenberger P 2014 Differential Code Bias Estimation Using Multi-GNSS Observations and Global Ionosphere Maps Navigation 61(3) 191-201.

Odijk D, Teunissen PJG 2013 Characterization of Between-Receiver GPS-Galileo Inter-System Biases and Their Effect on Mixed Ambiguity Resolution, GPS Solutions 17(4) 521-533. 
Phelts RE 2007 Range Biases on Modernized GNSS Codes, European Navigation Conference GNSS/TimeNav, 29 May 29 - 1 June 2007, Geneva, Switzerland.

Reussner N, Wanninger L 2011 GLONASS Inter-frequency Biases and Their Effects on RTK and PPP Carrier-phase Ambiguity Resolution, Proc. of ION GNSS 2011 Portland OR 712-716.

Schaer S, Dach R 2010 Biases in GNSS Analysis, IGS Workshop, Newcastle, England, 28 June - 2 July 2010.

Steigenberger P, Hugentobler U, Loyer S, Perosanz F, Prang L, Dach R, Uhlemann M, Gendt G, Montenbruck O 2014 Galileo Orbit and Clock Quality of the IGS Multi-GNSS Experiment Advances in Space Research 55(1) 269-281.

Tuka A, El-Mowafy A 2013 Performance Evaluation of Different Troposphere Delay Models and Mapping Functions, Measurement 46(2) 928-937.

Wen Z, Henkel P, and C Günter 2011 Reliable Estimation of Phase Biases of GPS Satellites with a Local Reference Network, Proceedings ELMAR, Zadar 14-16 Sept. 2011321 - 324.

Wübbena G, Schmitz M, Bagge A 2009 Some Thoughts on Satellite Induced Phase Shifts aka "the L2C Quarter Cycle Problem" and the Impact on RINEX and RTCM, Geo++ White Paper, accessed online on $4^{\text {th }}$ Dec 2014, http://www.geopp.de /media/docs/pdf/geopp_phase_shift_12c.pdf.

Wübbena, G. Schmitz, M. and A. Bagge 2014 PPP with Ambiguity Resolution (AR) Using RTCM-SSR, IGS Workshop Pasadena CA June 23-27 2014. 